\title{
Amino acid derivatives. Part 5. Synthesis and anti-HIV activity of new sebacoyl precursor derived thioureido-amino acid and phthalimide derivatives
}

\author{
Najim A. Al-Masoudi, ${ }^{a *}$ Nahed Al-Haidery, ${ }^{a}$ Naeem T. Failia and C. Pannecouque ${ }^{b}$ \\ ${ }^{a}$ Department of Chemistry, College of Science, University of Basrah, Basrah, Iraq \\ ${ }^{b}$ Rega Institute for Medical Research, Katholieke Universiteit Leuven, B-3000 Leuven, Belgium \\ E-mail: najim.al-masoudi@gmx.de
}

DOI: http://dx.doi.org/10.3998/ark.5550190.0011.918

\begin{abstract}
A series of sebacoyl $N, N$-bis(substituted-alkyl-2-thioureido)alkyl carboxylic acid or ester derivatives 3-12 bearing an amino acid ester residue were prepared by a one-pot sequential reaction of sebacoyl chloride 1 with $\mathrm{NH}_{4} \mathrm{SCN}$ and amino acid or their ester hydrochlorides. Analogously, the sebacoyl-phthalimido derivatives $\mathbf{1 6}$ and $\mathbf{1 7}$ were prepared from treatment of $\mathbf{1}$ with phthalimide precursors. Treatment of 5 and 7 with $\mathrm{Br}_{2}$ in acetone furnished the iminothiazole analogues 18 and 19, respectively. Compounds 5, 6, 8-11 and 16 have been selected for their inhibitory activity screening against HIV-1 and HIV-2 in MT-4 cells.
\end{abstract}

Keywords: Amino acids, anti-HIV activity, phthalimide, sebacoyl chloride

\section{Introduction}

HIV-1 reverse transcriptase is a key enzyme in the HIV replication as well as a key target for developing anti-HIV drugs. Two types of reverse transcriptase inihibitors have been developed $^{1,2}$ : nucleoside reverse transcriptase inihibitors (NRTIs) and non-nucleoside reverse transcriptase inhibitors (NNRTI). Three NNRTIs, nevirapine, ${ }^{3}$ delaviridine, ${ }^{4}$ and efavirenz ${ }^{5}$ have been approved by FDA for the treatment of HIV infection. However, significant resistance has been developed against the current NNRTI and there is an urgent need to develop new anti-HIV agents that are effective against these resistance mutants. ${ }^{6,7} \mathrm{We}$ have reported recently the synthesis of new nitroimidazoles with remarkable anti-HIV activity ${ }^{8-12}$ as NNRTIs candidates. Several heterocyclic thioureas have been reported as a new class of potent non-nucleoside reverse transcriptase inhibitors (NNRTIs) such as phenethylthiazolyl-thiourea (PETT) derivatives. ${ }^{13-16}$ Uckun et al. ${ }^{17}$ described the synthesis of a series of thiazole thioureas with alkyl, aryl, heteroaryl substituents as newly identified NNRTI of HIV, including mutant strains of HIV, 
and effective in the treatment of multi-drug resistant HIV infection. The synthesis of biologically active amino acid coupled derivatives was considered to be of a major interest. ${ }^{18-21}$ Recently, Fathalla et al. ${ }^{22,23}$ reported new quinazoline thioureas derivatives bearing an amino acid ester residue based on domino reaction of $N$-(2-cyanophenyl)benzimidoyl isothioyanate with amino acid methyl ester hydrochlorides.

In continuation of our work on amino acid derivatives, ${ }^{24-27}$ we described here the development of a new series of thioureas bearing amino acids or their ester analogues which can be used as potent NNRTI's
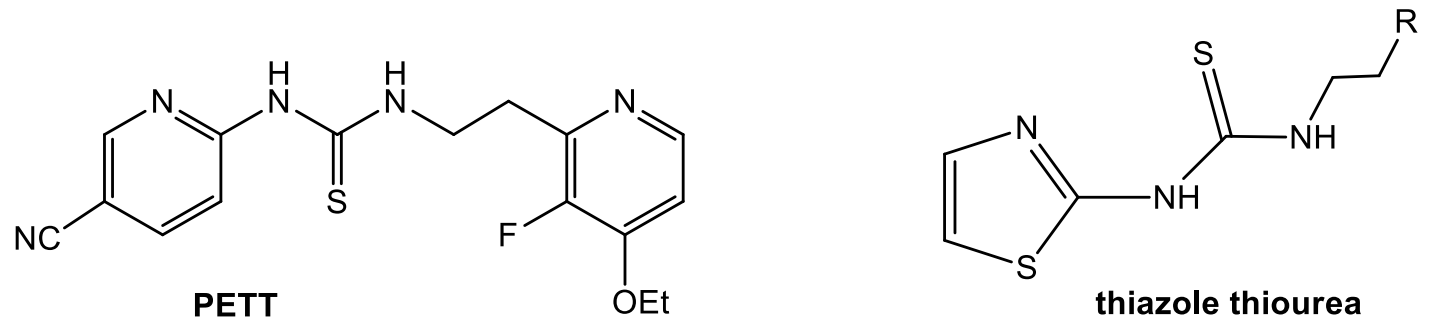

\section{Results and Discussion}

In our present work, sebacoyl chloride (octane-1,8-dicarboxylic acid dichloride) $\mathbf{1}$ has been selected as a spacer building block ${ }^{28}$ for the synthesis of new derivatives of sebacoyl- $N, N-$ bis(substituted-alkyl-2-thioureido)alkyl carboxylic acid or ester aiming for evaluation of their anti-HIV activity. Koenig et al. $^{29}$ have used 1 for the synthesis of 3,3,3',3'-tetraethyl-1,1'sebacoyl-bis(thiourea) and other analogues via the sebacoyl dithiocyanate derivative 2. Compound $\mathbf{2}$ was the key intermediate for synthesis of the compounds investigated in our work. Thus, treatment of $\mathbf{1}$ with $\mathrm{NH}_{4} \mathrm{SCN}$ in acetone, following Kabbani approach, ${ }^{30}$ afforded 2 which was directly treated with the desired amino acid derivatives to give, after purification, the sebacoyl-thioureido-amino acid derivatives in $63-86 \%$ yield. The synthetic reactions are summarized in scheme 1 .

The structures of 3-12 were determined by their ${ }^{1} \mathrm{H},{ }^{13} \mathrm{C}$ NMR and by mass spectra. The sebacoyl protons showed almost a similar pattern. $\mathrm{H}-7$ and $\mathrm{H}-14$ protons appeared as multiplets in the region $\delta$ 2.61-1.78 ppm, while $\mathrm{H}-8$ and $\mathrm{H}-13$ proton signals are oriented as multiplets in the region $\delta$ 1.81-1.45 ppm. H-9 - H-12 were appeared as multiplets in the region $\delta \mathrm{H}-2$ of the amino acid moieties are oriented in the region $\delta$ with different multiplicities, depending on the functional group adjacent to $\mathrm{H}-2$. The other protons of the amino acids or esters were fully analyzed. The ${ }^{13} \mathrm{C}$ NMR spectra of 3-12 contained almost similar resonance signals of the sebacoyl C-7 - C-14 and thioureido carbon atoms. The chemical shifts between $\delta 188.8$ and $184.25 \mathrm{ppm}$ were assigned to $\mathrm{C}=\mathrm{S}$ carbon atom of the thioureido moiety (C-4), while the resonances in the range of $\delta 177.7-174.1 \mathrm{ppm}$ were assigned to the carbonyl groups of the $\mathrm{CSNHCO}$ residues. $\mathrm{C}-2$ of the amino acid moieties $\left[\mathrm{CH}-\mathrm{CO}_{2} \mathrm{H}(\mathrm{Me}, \mathrm{Et})\right]$ appeared in the region $\delta$ 
66.7-55.9 ppm. The sebacoyl carbon atoms C-7 and C-14 are oriented in the region $\delta 38.0-35.3$, while C-8 and C-13 were appeared in the region $\delta$ 26.4-25.0 ppm. The signals between $\delta$ $\square 31.5$ and $24.7 \mathrm{ppm}$ were attributed to $\mathrm{C}-9$ and $\mathrm{C}-12$.

The proton spin system of $\mathbf{1 1}$ was further identified from DFQ-COSY ${ }^{31}$ spectrum, where the doublet of $\mathrm{H}^{2}$ alanin at $\delta 3.41 \mathrm{ppm}$ was found to correlate with $\mathrm{CO}_{2} \mathrm{H}-\mathrm{C}^{2}$ alanin- $\mathrm{H}$ ) at $\delta_{\mathrm{C}} 55.9 \mathrm{ppm}$. In the ${ }^{1} \mathrm{H}$ NMR (HMQC) ${ }^{32}$ spectrum of 11, the multiplets at $\delta_{\mathrm{H}} 2.25$ and $1.51 \mathrm{ppm}$ of carbon atoms resonating at $\delta_{\mathrm{C}} 35.7$ and $25.5 \mathrm{ppm}$ were assigned to $\left(\mathrm{CH}_{2}-7+\mathrm{CH}_{2}-14\right)$ and $\left(\mathrm{CH}_{2}-8+\mathrm{CH}_{2}-13\right)$, respectively, by spin decoupling experiment. Similary, the methylene protons $\left(\mathrm{CH}_{2}-9-\mathrm{CH}_{2}-12\right)$ at $\delta_{\mathrm{H}} 1.29 \mathrm{ppm}$ and their carbon atoms $(\mathrm{C}-9+\mathrm{C}-12)\left(\delta_{\mathrm{C}} 30.2\right)$ and $(\mathrm{C}-10+\mathrm{C}-11)\left(\delta_{\mathrm{C}} 28.2 \mathrm{ppm}\right)$ have been identified. From the gradient selected $\mathrm{HMBC}^{33}$ spectrum of 11, $\mathrm{H}^{2}$ alanin proton at $\delta_{\mathrm{H}}$ $3.41 \mathrm{ppm}$ showed two ${ }^{2} J_{\mathrm{C}, \mathrm{H}}$ couplings: one with $\mathrm{CO}_{2} \mathrm{H}$ at $\delta_{\mathrm{C}} 173.0 \mathrm{ppm}$, and the other with $\mathrm{Me}_{\text {alanin }}$ at $\delta_{\mathrm{C}} 17.3 \mathrm{ppm}$.
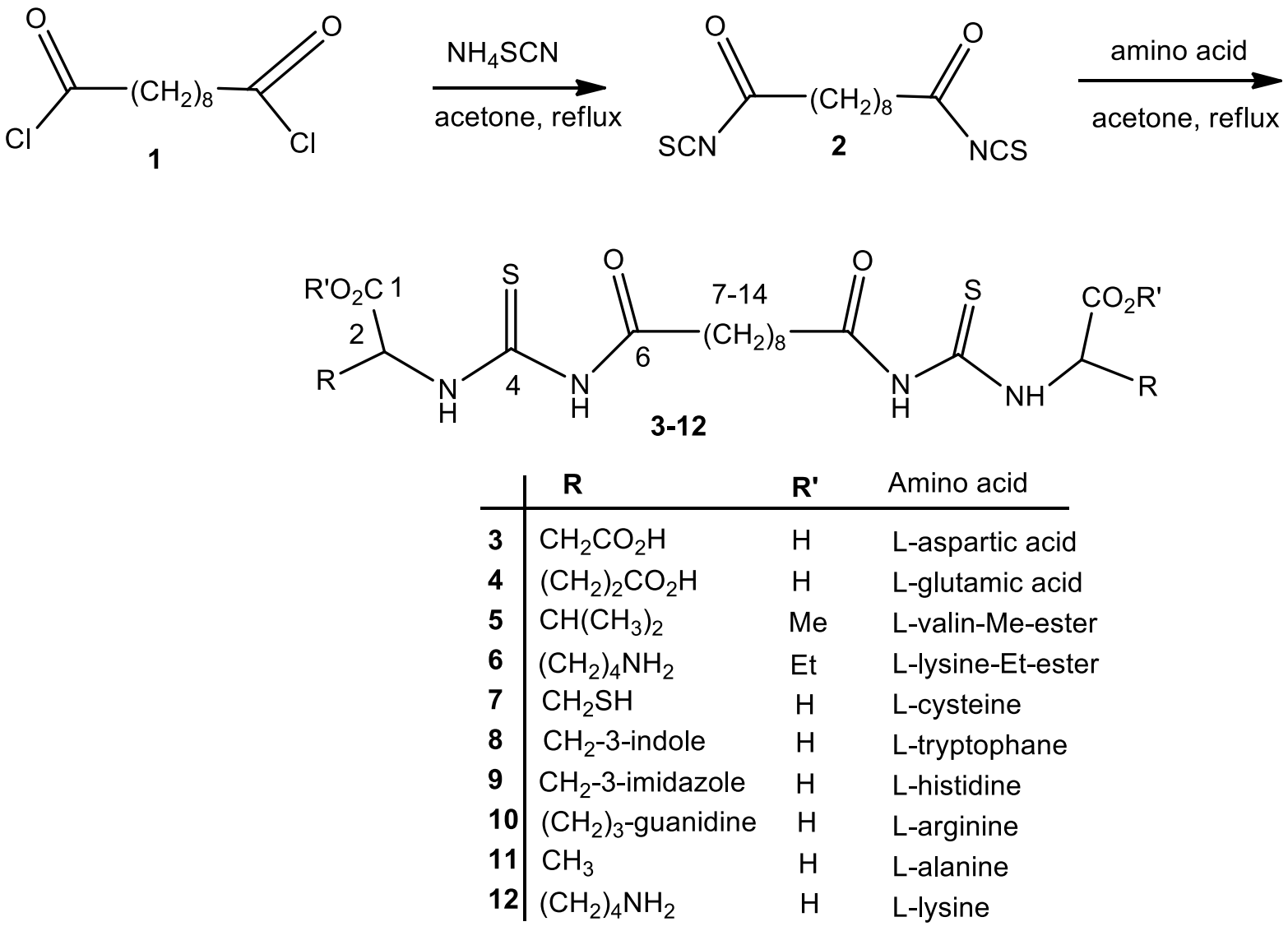

Scheme 1. Synthesis of sebacoyl N,N-bis(substituted-alkyl-2-thioureido)alkyl carboxylic acid or ester derivatives.

Next, sebacoyl chloride $\mathbf{1}$ was treated with phthalimide $\mathbf{1 3}$ or $\mathrm{N}$ (phthalimido)methylmagnesium bromide 15, prepared from hydroxymethyl-phthalimide $\mathbf{1 4},{ }^{34}$ in refluxing acetone afforded after purification the sebacoyl- $N, N$-bis-phthalimide $\mathbf{1 6}$ and the 
methylphthalimide analogue $\mathbf{1 7}$ in 83 and $86 \%$ yield, respectively (scheme 2). The structures of 16 and 17 were assigned by the ${ }^{1} \mathrm{H},{ }^{13} \mathrm{C}$ NMR and mass spectra. The ${ }^{1} \mathrm{H}$ NMR spectra showed rather similar patterns for the sebacoyl $\left(\mathrm{CH}_{2}\right)$ protons for those of 3-12, meanwhile, the singlet at $\delta 4.96 \mathrm{ppm}$ was attributed to the ethylene group adjacent to the phthalimide precursor. In the ${ }^{13} \mathrm{C}$ NMR spectra of $\mathbf{1 6}$ and 17, the higher-field resonances at $\delta 172.1$ and $174.5 \mathrm{ppm}$ were attributed to $\mathrm{C}=\mathrm{O}$ group of the sebacoyl moiety, while the resonances at $\delta 167.3$ and $167.4 \mathrm{ppm}$ were assigned to $\mathrm{C}=\mathrm{O}$ of the phthalimide residue.
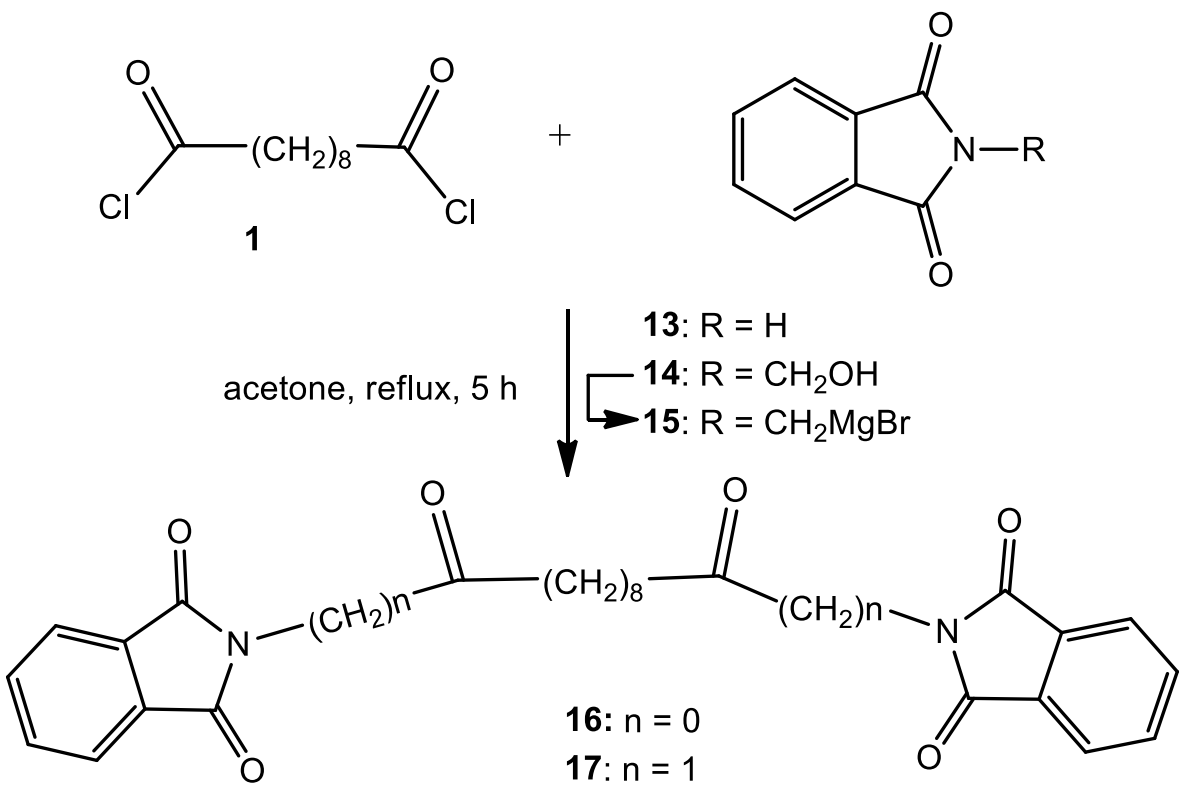

Scheme 2. Synthesis of sebacoyl-N,N-bis-phthalimide and methyl analogue.

Further, our work was modified by selecting 5 and $\mathbf{7}$ as precursors for the synthesis of new analogues of sebacoyl-2-imino-thiazole. Thus, treatment of $\mathbf{5}$ and $\mathbf{7}$ with acetone and bromine under reflux led to cyclization of the thioureido residue furnishing the 2-imino-thiazole derivatives 18 and $\mathbf{1 9}$ in 65 and $71 \%$ yields, respectively (Scheme 3). The structures of $\mathbf{1 8}$ and $\mathbf{1 9}$ were determined from their ${ }^{1} \mathrm{H}-,{ }^{13} \mathrm{C}$ NMR and mass spectra. The sebacoyl protons showed rather similar pattern for the sebacoyl $\left(\mathrm{CH}_{2}\right)$ protons for those of $\mathbf{1 6}$ and 17. The singlets at $\delta 5.87$ and $5.92 \mathrm{ppm}$ were assigned to $\mathrm{H}-5$ of the thiazole ring, respectively, while the singlets at $\delta 1.70$ and $1.68 \mathrm{ppm}$ were attributed to the methyl groups at $\mathrm{C}-4$ of the thiazole moiety. In the ${ }^{13} \mathrm{C}$ NMR of 18 and 19, the resonances at $\delta 163.8$ and $163.6 \mathrm{ppm}$, were attributed to $\mathrm{C}=\mathrm{N}(\mathrm{C}-2)$, respectively, whereas the signals at $\delta 132.9$ and $132.7 \mathrm{ppm}$ were assigned to C-4, respectively. C-5 were oriented between $\delta 100.1$ and $99.8 \mathrm{ppm}$, respectively. The structures of $\mathbf{1 8}$ and $\mathbf{1 9}$ were further confirmed by the gradient ${ }^{33}$ selected HMBC spectra. $\mathrm{H}-5$ of the thiazole ring at $\delta_{\mathrm{H}} 5.87$ and 5.92 ppm showed ${ }^{2} J_{\mathrm{C}, \mathrm{H}}$ couplings with C-4 of the thiazole ring at $\delta_{\mathrm{C}} 132.9$ and $132.7 \mathrm{ppm}$, as well as ${ }^{3} J_{\mathrm{C}, \mathrm{H}}$ couplings with $\mathrm{C}=\mathrm{N}(\mathrm{C}-2)$ of the thiazole ring at $\delta_{\mathrm{C}} 163.8$ and $163.6 \mathrm{ppm}$, respectively. 

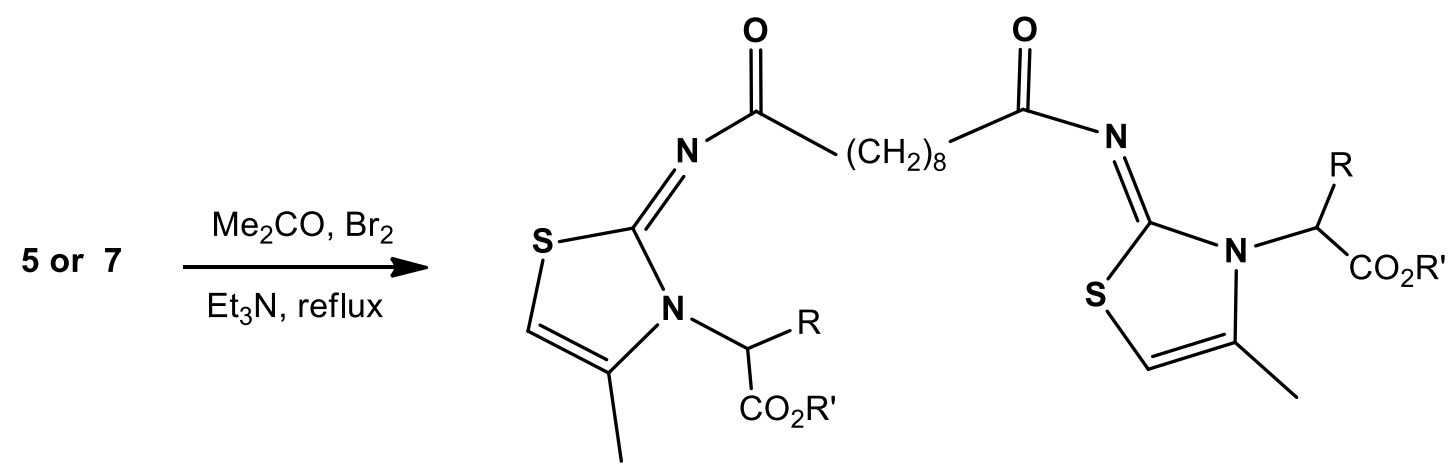

\begin{tabular}{l|lll} 
& $\mathbf{R}$ & $\mathbf{R}^{\prime}$ & Amino acid \\
\hline 18 & $\left(\mathrm{CH}(\mathrm{Me})_{2}\right.$ & $\mathrm{Me}$ & L-valin-Me-ester \\
19 & $\mathrm{CH}_{2} \mathrm{SH}$ & $\mathrm{H}$ & L-cystein
\end{tabular}

Scheme 3. Synthesis of sebacoyl N,N-bis-methyl-(alkyl-2-imino-thiazol-3-yl)butanoate or propanoic acid.

\section{In vitro anti-HIV assay}

Compounds 5, 6, 8-11 and 16 were tested for their in vitro anti-HIV-1 (strain III $_{\mathrm{B}}$ ) and HIV-2 (strain ROD) activity in human T-lymphocyte (MT-4) cells, based on MTT assay. ${ }^{35}$ None of the new compounds were found to inhibit HIV-1 and HIV-2 replication, in vitro, at $\mathrm{IC}_{50}$ lower than the $\mathrm{CC}_{50}$ in comparison to the antiviral agents Nevirapine (BOE/BIRG587) ${ }^{36}$ and azidothymidine (AZT) ${ }^{37}$ In conclusion, the above data showed no selective anti-HIV activity.

\section{Experimental section}

General. Melting points are uncorrected and were measured on a Büchi melting point apparatus B-545 (Büchi Labortechnik AG, Switzerland). Microanalytical data were obtained with a Vario, Elemental apparatus (Shimadzu, Japan). NMR spectra were recorded on 400 and $600 \mathrm{MHz}\left({ }^{1} \mathrm{H}\right)$ and on $150.91 \mathrm{MHz}\left({ }^{13} \mathrm{C}\right)$ spectrometers (Bruker, Germany) with TMS as internal standard and on the $\delta$ scale in ppm. Signal assignments for protons were identified by selective proton decoupling or by COSY spectra. Heteronuclear assignments were verified by ${ }^{1} \mathrm{H}^{-13} \mathrm{C}$ COSY, or HMBC experiments. Mass spectra were recorded at $70 \mathrm{eV}$ on EI. TLC plates 60 F254 were purchased from Merck.

General procedure of preparation of sebacoyl $N, N$-bis(substituted-alkyl-2-thioureido)alkyl carboxylic acid or ester derivatives (3-12)

A solution of sebacoyl chloride $(0.72 \mathrm{~g}, 3.0 \mathrm{mmol})$ and $\mathrm{NH}_{4} \mathrm{SCN}(0.46 \mathrm{~g}, 6.0 \mathrm{mmol})$ in acetone $(20 \mathrm{~mL})$ was heated under reflux for $1 \mathrm{~h}$. After 
cooling and filtration, a solution of the desired free amino acid or the ester analogue (6.0 mmol) in dry acetone $(15 \mathrm{~mL})$ was added and the mixture was heated under reflux for $6 \mathrm{~h}$. After cooling, an excess of crushed ice was pouted on the mixture with vigrous stirring. The resulting result was collected, washed with acetone and recrystallized from EtOH or DMF-ether.

Sebacoyl- $\boldsymbol{N}, \boldsymbol{N}$-bis(2-thioureido)succinic acid (3). From L-aspartic acid (0.80 g), Yield: $1.0 \mathrm{~g}$ $(61 \%)$; mp 235-236 ${ }^{\circ} \mathrm{C} .{ }^{1} \mathrm{H}$ NMR (DMSO- $\left.d_{6}\right): \delta 3.77\left(\mathrm{dd}, 2 \mathrm{H}, J_{\mathrm{H} 2 \text {-aspar,H3a-aspar. }}=3.4 \mathrm{~Hz}, J_{\mathrm{H} 2-}\right.$ aspar.,H3b-aspar. $=11.4 \mathrm{~Hz}, \mathrm{CO}_{2} \mathrm{H}-H^{2}$ aspar); 3.17 (br s., $\left.1 \mathrm{H}, \mathrm{NH}\right) ; 2.75\left(\mathrm{dd}, 1 \mathrm{H}, J_{\mathrm{H} 3 \mathrm{a}, \mathrm{H} 3 \mathrm{~b} \text {-aspar. }}=15.0 \mathrm{~Hz}\right.$, $H^{3 \mathrm{a}}$ aspar); $2.72\left(\mathrm{dd}, 1 \mathrm{H}, H^{3 \mathrm{~b}}\right.$ aspar); 2.61 (m, 2H, $\left.\mathrm{CH}_{2}-7+\mathrm{CH}_{2}-14\right) ; 1.69$ (m, 4H, $\mathrm{CH}_{2}-8+\mathrm{CH}_{2}-13$ ): 1.29-1.23 (m, 8H, $\left.\mathrm{CH}_{2}-9+\mathrm{CH}_{2}-10+\mathrm{CH}_{2}-11+\mathrm{CH}_{2}-12\right) .{ }^{13} \mathrm{C} \mathrm{NMR}$ (DMSO-d $\left.d_{6}\right): \delta 184.9(\mathrm{C}=\mathrm{S})$; $174.3\left(\mathrm{CSNHCO}+\mathrm{CO}_{2} \mathrm{H}\right) ; 137.1,127.3,123.3,119.8,11.2,109.9\left(\mathrm{C}_{\text {trypt. }}\right) ; 66.7\left(\mathrm{CO}_{2} \mathrm{H}-\mathrm{CH}\right)$; 35.5 (C-7 + C-14); 31.5 (C10 + C-11); 29.0 (C-9 + C-12); 26.4 (C-8 + C-13). Anal. calc. for $\mathrm{C}_{20} \mathrm{H}_{30} \mathrm{~N}_{4} \mathrm{O}_{10} \mathrm{~S}_{2}$ (550.6): C, 43.63; H, 5.49; N, 10.18. Found: C, 43.34; H, 5.41; N, 9.89. MS: m/z (FAB) $551[\mathrm{M}+\mathrm{H}]^{+}$.

Sebacoyl- $\boldsymbol{N}, \boldsymbol{N}$-bis(2-thioureido)-L-glutamic acid (4). From L-glutamic acid (0.88 g). Yield: 1.47 g (85\%); mp 195-196 ${ }^{\circ} \mathrm{C} .{ }^{1} \mathrm{H}$ NMR (DMSO- $d_{6}$ ): $\delta 11.08$ (br s., $2 \mathrm{H}, \mathrm{CO}_{2} \mathrm{H}$ ); 3.86 (br s., $1 \mathrm{H}$, $\mathrm{NH}) ; 3.59\left(\mathrm{dd}, 2 \mathrm{H}, J_{\mathrm{H} 2 \text {-glutamic,H3a-glutamic }}=3.5 \mathrm{~Hz}, J_{\mathrm{H} 2 \text {-glutamic, } \mathrm{H} 3 \mathrm{~b} \text {-glutamic }}=11.5 \mathrm{~Hz}, \mathrm{CO}_{2} \mathrm{H}-\mathrm{H}^{2}\right.$ glutamic ); $2.29\left(\mathrm{~m}, 4 \mathrm{H}, \mathrm{H}^{4 \mathrm{a}, \mathrm{b}}\right.$ glutamic $) ; 2.14\left(\mathrm{~m}, 4 \mathrm{H}, \mathrm{CH}_{2}-7+\mathrm{CH}_{2}-14\right): 2.11\left(\mathrm{~m}, 4 \mathrm{H}, \mathrm{H}^{3 \mathrm{a}, \mathrm{b}}\right.$ glutamic $) ; 1.81\left(\mathrm{CH}_{2}-8\right.$ $\left.+\mathrm{CH}_{2}-13\right) ; 1.31-1.21\left(\mathrm{~m}, 8 \mathrm{H}, \mathrm{CH}_{2}-9+\mathrm{CH}_{2}-10+\mathrm{CH}_{2}-11+\mathrm{CH}_{2}-12\right) .{ }^{13} \mathrm{C}$ NMR (DMSO-d $): \delta$ $185.7(\mathrm{C}=\mathrm{S}) ; 177.5\left(\mathrm{CO}_{2} \mathrm{H}\right) ; 174.7\left(\mathrm{CSNHCO}+\mathrm{CO}_{2} \mathrm{H}\right) ; 61.9\left(\mathrm{CO}_{2} \mathrm{H}-\mathrm{CH}\right) ; 35.7(\mathrm{C}-7+\mathrm{C}-14)$; $30.8\left(\mathrm{C} 10+\mathrm{C}-11+\mathrm{C}^{4}\right.$ glutamic $) ; 29.2(\mathrm{C}-9+\mathrm{C}-12) ; 26.4\left(\mathrm{C}-8+\mathrm{C}-13+\mathrm{C}^{3}\right.$ glutamic $)$. Anal. calc. for $\mathrm{C}_{22} \mathrm{H}_{34} \mathrm{~N}_{4} \mathrm{O}_{10} \mathrm{~S}_{2}$ (578.66): C, 45.66; H, 5.92; N, 9.68. Found: C, 45.35; H, 5.87; N, 9.42. MS: m/z (FAB) $579[\mathrm{M}+\mathrm{H}]^{+}$.

Sebacoyl $\boldsymbol{N}, \boldsymbol{N}$-bis-methyl(2-thioureido)-3-methylbutanoate (5). From L-valine methyl ester (0.79 g). Yield: $1.04 \mathrm{~g}(63 \%)$; mp 260-262 ${ }^{\circ} \mathrm{C} .{ }^{1} \mathrm{H}$ NMR (DMSO- $\left.d_{6}\right): \delta 8.01$ (br s., $\left.1 \mathrm{H}, \mathrm{NH}\right) ; 3.71$ $\left(\mathrm{s}, 3 \mathrm{H}, \mathrm{CO}_{2} \mathrm{Me}\right) ; 3.46\left(\mathrm{dd}, 2 \mathrm{H}, J_{2,3(\text { valin })}=7.5 \mathrm{~Hz}, 2 \mathrm{xH}_{\text {valin }}^{2}\right) ; 2.76\left(\mathrm{~m}, 2 \mathrm{H}, 2 \mathrm{xH}^{3}{ }_{\text {valin }}\right) ; 2.01(\mathrm{~m}, 4 \mathrm{H}$, $\left.\mathrm{CH}_{2}-7+\mathrm{CH}_{2}-14\right) ; 1.51$ (m, $\left.4 \mathrm{H}, \mathrm{CH}_{2}-8+\mathrm{CH}_{2}-13\right) ; 1.32$ (m, $\left.4 \mathrm{H}, \mathrm{CH}_{2}-9+\mathrm{CH}_{2}-12\right) ; 1.24\left(\mathrm{CH}_{2}-10\right.$ $\left.+\mathrm{CH}_{2}-11\right) ; 1.08\left(\mathrm{~m}, 12 \mathrm{H}, 4 \mathrm{xCH}_{3}\right) \cdot{ }^{13} \mathrm{C} \mathrm{NMR}\left(\mathrm{DMSO}-d_{6}\right): \delta 187.1(\mathrm{C}=\mathrm{S}) ; 174.3(\mathrm{CSNHCO}) ;$ 170.8 (COEt); $61.8\left(\mathrm{CO}_{2} \mathrm{Me}-\mathrm{CH}\right) ; 52.1\left(\mathrm{CO}_{2} \mathrm{Me}\right) ; 37.4(\mathrm{C}-7+\mathrm{C}-14) ; 31.4\left(\mathrm{C}^{3}\right.$ valin $) ; 29.8(\mathrm{C}-9+$ $\mathrm{C}-10+\mathrm{C}-11+\mathrm{C}-12) ; 26.1(\mathrm{C}-8+\mathrm{C}-13) ; 18.3\left(\mathrm{CH}_{3}\right)$. Anal. calc. for $\mathrm{C}_{24} \mathrm{H}_{42} \mathrm{~N}_{4} \mathrm{O}_{6} \mathrm{~S}_{2}(546.74)$ : C, 52.72; H, 7.74; N, 10.25. Found: C, 52.50; H, 7.68; N, 10.02. MS: m/z (FAB) $547[\mathrm{M}+\mathrm{H}]^{+}$.

Sebacoyl- $\boldsymbol{N}, \boldsymbol{N}$-bis-ethyl-(6-amino-2-thioureido)hexanoate (6). From L-lysine ethyl ester dihydrochloride (1.48 g). Yield: $2.14 \mathrm{~g}$ (78\%), mp 108-110 ${ }^{\circ} \mathrm{C} .{ }^{1} \mathrm{H}$ NMR (DMSO- $\left.d_{6}\right): \delta 8.73$, br s., $2 \mathrm{H}, 2 \mathrm{xNH}$ ); 8.23 (br s., $2 \mathrm{H}, 2 \mathrm{xNH}$ ); 4.22 (q, $\left.4 \mathrm{H}, \mathrm{J}=7.0 \mathrm{~Hz}, 2 \mathrm{xOCH} \mathrm{CH}_{3}\right) ; 3.92$ (t, 2H, J $\mathrm{H}_{2}-$ lysin,H3-a,b) $=6.1 \mathrm{~Hz}, 2 \mathrm{xH}^{2}{ }_{\text {lysin }}$ ); 2.72 (br s., $\left.8 \mathrm{H}, 2 \mathrm{xCH}_{2}-\mathrm{NH}_{2}+2 \mathrm{xNH}_{2}\right) ; 1.82-1.77$ (m, $8 \mathrm{H}, 2 \mathrm{xCH}_{2^{-}}$ $\left.3_{\text {lysin }}+\mathrm{CH}_{2}-7+\mathrm{CH}_{2}-14\right) ; 1.59\left(\mathrm{~m}, 6 \mathrm{H}, 2 \mathrm{xCH}_{2}-5_{\text {lysin }}+2 \mathrm{xNH}\right) ; 1.47$ (m, 4H, $\left.\mathrm{CH}_{2}-8+\mathrm{CH}_{2}-13\right) ; 1.38$ (m, $\left.4 \mathrm{H}, \mathrm{CH}_{2}-9+\mathrm{CH}_{2}-12\right) ; 1.23\left(\mathrm{~m}, 10 \mathrm{H}, 2 \mathrm{xOCH}_{2} \mathrm{CH}_{3}+\mathrm{CH}_{2}-10+\mathrm{CH}_{2}-11\right) .{ }^{13} \mathrm{C}$ NMR (DMSO$\left.d_{6}\right): \delta 187.9(\mathrm{C}=\mathrm{S}) ; 174.1(\mathrm{CSNHCO}) ; 170.6(\mathrm{COEt}) ; 61.7\left(\mathrm{OCH}_{2} \mathrm{CH}_{3}+\mathrm{CO}_{2} \mathrm{Et}-\mathrm{CH}\right) ; 51.1$ $\left(\mathrm{CH}_{2} \mathrm{NH}_{2}\right) ; 37.8(\mathrm{C}-7+\mathrm{C}-14) ; 29.2\left(\mathrm{C}^{3}\right.$ lysin $\left.+\mathrm{C}-9+\mathrm{C}-10+\mathrm{C}-11+\mathrm{C}-12\right) ; 26.0(\mathrm{C}-8+\mathrm{C}-13)$; $21.1\left(\mathrm{C}^{4}\right.$ lysin); $13.9\left(\mathrm{OCH}_{2} \mathrm{CH}_{3}\right)$. Anal. calc. for $\mathrm{C}_{28} \mathrm{H}_{52} \mathrm{~N}_{6} \mathrm{O}_{6} \mathrm{~S}_{2}(632.88): \mathrm{C}, 53.14 ; \mathrm{H}, 8.28 ; \mathrm{N}$, 13.28. Found: C, 52.94; H, 8.19; N, 13.05. MS: m/z (FAB) $633[\mathrm{M}+\mathrm{H}]^{+}$. 
Sebacoyl- $N, N$-bis(4-mercapto-2-thioureido)butanoic acid (7). From L-cysteine $(0.73 \mathrm{~g})$. Yield: $1.14 \mathrm{~g}(72 \%) ; \mathrm{mp} 215-217^{\circ} \mathrm{C} .{ }^{1} \mathrm{H}$ NMR (DMSO- $\left.d_{6}\right): \delta 4.03\left(\mathrm{dd}, 2 \mathrm{H}, J_{\mathrm{H} 2 \text {-cystein,H3-a) }}=7.1\right.$ $\left.\mathrm{Hz}, J_{\mathrm{H} 2 \text {-cystein,H3-b) }}=14.2 \mathrm{~Hz} 2 \mathrm{xH}_{\text {cystein }}\right) ; 3.16$ (br s., $4 \mathrm{H}, 2 \mathrm{xH}^{3 \mathrm{a}}$ cystein $+2 \mathrm{xH}^{3 \mathrm{~b}}$ cystein); 2.32 (m, 4H, $\left.+\mathrm{CH}_{2}-7+\mathrm{CH}_{2}-14\right) ; 1.52\left(\mathrm{~m}, 4 \mathrm{H}, \mathrm{CH}_{2}-8+\mathrm{CH}_{2}-13\right)$ ); $1.29\left(\mathrm{~m}, 4 \mathrm{H}, \mathrm{CH}_{2}-9+\mathrm{CH}_{2}-12\right) ; 1.21$ (m, $\left.4 \mathrm{H}, \mathrm{CH}_{2}-10+\mathrm{CH}_{2}-11\right) .{ }^{13} \mathrm{C} \mathrm{NMR}\left(\mathrm{DMSO}-d_{6}\right): \delta 188.8(\mathrm{C}=\mathrm{S}) ; 175.7(\mathrm{CSNHCO}) ; 174.9\left(\mathrm{CO}_{2} \mathrm{H}\right)$; $63.9\left(\mathrm{CO}_{2} \mathrm{H}-\mathrm{CH}\right) ; 36.4(\mathrm{C}-7+\mathrm{C}-14) ; 32.0(\mathrm{C}-10+\mathrm{C}-11) ; 28.9(\mathrm{C}-9+\mathrm{C}-12) ; 27.2\left(\mathrm{CH}_{2} \mathrm{SH}\right)$; 25.0 (C-8 + C-13). Anal. calc. for $\mathrm{C}_{18} \mathrm{H}_{30} \mathrm{~N}_{4} \mathrm{O}_{6} \mathrm{~S}_{4}$ (526.71): C, 41.05; H, 5.74; N, 10.64. Found: C, 40.89; H, 5.75; N, 10.43. MS: m/z (FAB) $527[\mathrm{M}+\mathrm{H}]^{+}$.

Sebacoyl- $N, N$-bis(2-thioureido-3-(indol-3-yl))propanoic acid (8). From L-tryptophane (1.23 g). Yield: $1.4 \mathrm{~g}(67 \%)$; mp 255-257 ${ }^{\circ} \mathrm{C} .{ }^{1} \mathrm{H}$ NMR (DMSO-d $): \delta 10.90\left(\mathrm{~s}, 1 \mathrm{H}, \mathrm{CO}_{2} \mathrm{H}\right) ; 7.20$ $\left(1 \mathrm{H}, \mathrm{d}, J_{2, \mathrm{NH}}=2.2 \mathrm{~Hz}, \mathrm{H}_{\text {trypt. }}^{2}\right) ; 7.56\left(\mathrm{~d}, 1 \mathrm{H}, J=7.8 \mathrm{~Hz}, \mathrm{H}_{\text {trypt }}^{7}\right) ; 7.34\left(\mathrm{~d}, 1 \mathrm{H},=8.0 \mathrm{~Hz}, \mathrm{H}_{\text {trypt }}^{4}\right.$; $7.07\left(\mathrm{t}, 1 \mathrm{H}, J=8.0 \mathrm{~Hz}, \mathrm{H}_{\text {trypt }}^{5}\right) ; 6.98\left(\mathrm{t}, 1 \mathrm{H}, J=7.8 \mathrm{~Hz}, \mathrm{H}_{\text {trypt }}^{6}\right) .3 .43\left(\mathrm{dd}, 2 \mathrm{H}, J_{2, \text { ch2a-trypt. }}=4.0 \mathrm{~Hz}\right.$, $\left.J_{2, \text { CH2b-trypt. }}=9.0 \mathrm{~Hz}, \mathrm{CO}_{2} \mathrm{H}-2 \mathrm{x} H_{\text {trypt. }}^{2}\right) ; 3.31\left(\mathrm{dd}, 1 \mathrm{H}, \mathrm{CH}_{2} \mathrm{a}-\right.$ trypt$) ; 2.93\left(\mathrm{dd}, 1 \mathrm{H}, J_{\mathrm{Ha}, \mathrm{Hb}-\text { trypt. }}=15.0\right.$ $\mathrm{Hz}, \mathrm{CH}_{2}$ b-trypt.); 2.33 (m, 4H, $\mathrm{CH}_{2}-7+\mathrm{CH}_{2}-14$ ); 1.69 (m, 4H, $\mathrm{CH}_{2}-8+\mathrm{CH}_{2}-13$ ): 1.29-1.23 (m, $\left.8 \mathrm{H}, \mathrm{CH}_{2}-9+\mathrm{CH}_{2}-10+\mathrm{CH}_{2}-11+\mathrm{CH}_{2}-12\right) \cdot{ }^{13} \mathrm{C}$ NMR (DMSO-d $): \delta 184.9(\mathrm{C}=\mathrm{S}) ; 174.3$ $\left(\mathrm{CSNHCO}+\mathrm{CO}_{2} \mathrm{H}\right) ; 137.1,127.3,123.3,119.8,111.2,109.9\left(\mathrm{C}_{\text {trypt. }}\right) ; 62.7\left(\mathrm{CO}_{2} \mathrm{H}-\mathrm{CH}\right) ; 35.5(\mathrm{C}-$ 7 + C-14); 31.5 (C-10 + C-11); 29.0 (C-9 + C-12); 26.4 (C-8 + C-13). Anal. calc. for $\mathrm{C}_{34} \mathrm{H}_{40} \mathrm{~N}_{6} \mathrm{O}_{6} \mathrm{~S}_{2}$ (692.85): C, 58.94; H, 5.82; N, 12.13. Found: C, 58.72; H, 4.09; N, 11.97. MS: $\mathrm{m} / \mathrm{z}(\mathrm{FAB}) 693[\mathrm{M}+\mathrm{H}]^{+}$.

Sebacoyl- $N, N$-bis(2-thioureido-3-(imidazol-4-yl))propanoic acid (9). From L-histidine (0.93 g). Yield: $1.43 \mathrm{~g}(80 \%)$; mp 240-242 ${ }^{\circ} \mathrm{C} .{ }^{1} \mathrm{H}$ NMR (DMSO- $\left.d_{6}\right): \delta 7.38$ (s, $1 \mathrm{H}, \mathrm{H}^{2}$ imidazol); $6.37\left(\mathrm{~s}, 1 \mathrm{H}, \mathrm{H}^{5}\right.$ imidazol); $3.64\left(\mathrm{dd}, 2 \mathrm{H}, J_{2}{ }^{\prime}, 3^{\prime}\right.$ a(histidin) $=7.5 \mathrm{~Hz}, J_{2}{ }^{\prime}, 3^{\prime}$ ba(histidin) $=13.5 \mathrm{~Hz} 2 \mathrm{xH}^{2}{ }_{\text {histidin }}$ ); 3.10 (m., 4H, 2xH $\mathrm{Ha}_{\text {hisidin }}+2 \mathrm{xH}^{3 \mathrm{~b}}$ histidin); 2.14 (m, 4H, $\left.+\mathrm{CH}_{2}-7+\mathrm{CH}_{2}-14\right) ; 1.63$ (m, 4H, $\mathrm{CH}_{2}-8+$ $\left.\mathrm{CH}_{2}-13\right) ; 1.28$ (m, 4H, $\left.\mathrm{CH}_{2}-9+\mathrm{CH}_{2}-12\right) ; 1.23$ (m, 4H, $\left.\mathrm{CH}_{2}-10+\mathrm{CH}_{2}-11\right) .{ }^{13} \mathrm{C}$ NMR (DMSO$\left.d_{6}\right): \delta 184.2(\mathrm{C}=\mathrm{S}) ; 177.7(\mathrm{CSNHCO}) ; 174.0\left(\mathrm{CO}_{2} \mathrm{H}\right) ; 135.4,\left(\mathrm{C}^{2}{ }_{\text {imidazol }}\right) ; 132.7\left(\mathrm{C}^{4}{ }_{\text {imidazol }}\right) ; 120.3$ $\left(\mathrm{C}^{5}\right.$ imidazol $) ; 61.9\left(\mathrm{CO}_{2} \mathrm{H}-\mathrm{CH}\right) ; 37.4(\mathrm{C}-7+\mathrm{C}-14) ; 29.9\left(\mathrm{C}-10+\mathrm{C}-11+\mathrm{C}^{3}{ }_{\text {imidazol }}\right): 28.2(\mathrm{C}-9+\mathrm{C}-$ 12); 26.0 (C-8 + C-13). Anal. calc. for $\mathrm{C}_{24} \mathrm{H}_{34} \mathrm{~N}_{8} \mathrm{O}_{6} \mathrm{~S}_{2}$ (597.71): C, 48.47; H, 5.76; N, 18.84. Found: C, 48.22; H, 5.66; N, 18.67. MS: m/z (FAB) $598[\mathrm{M}+\mathrm{H}]^{+}$.

Sebacoyl- $N, N$-bis(2-thioureido-5-guanidino)pentanoic acid (10). From L-arginine (1.04 g). Yield: $1.64 \mathrm{~g}(86 \%)$; mp 120-122 ${ }^{\circ} \mathrm{C} .{ }^{1} \mathrm{H}$ NMR (DMSO- $\left.d_{6}+\mathrm{D}_{2} \mathrm{O}\right): \delta 3.69\left(\mathrm{dd}, 2 \mathrm{H}, J_{\mathrm{H} 2 \text {-arginin, H3a- }}\right.$

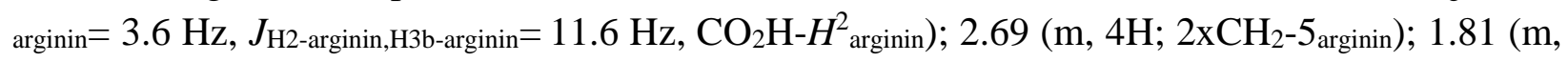
$\left.4 \mathrm{H}, 2 \mathrm{xCH}_{2}-3_{\text {arginin }}\right) ; 1.91\left(\mathrm{~m}, 4 \mathrm{H}, \mathrm{CH}_{2}-7+\mathrm{CH}_{2}-14\right) ; 1.61\left(\mathrm{~m}, 8 \mathrm{H}, 2 \mathrm{xCH}_{2}-4\right.$ arginin $+\mathrm{CH}_{2}-8+\mathrm{CH}_{2}-$ 13); 1.31-1.27 (m, 8H, CH $\left.2-9+\mathrm{CH}_{2}-10+\mathrm{CH}_{2}-11+\mathrm{CH}_{2}-12\right) .{ }^{13} \mathrm{C} \mathrm{NMR}$ (DMSO-d $\left.6+\mathrm{D}_{2} \mathrm{O}\right): \delta$ $184.7(\mathrm{C}=\mathrm{S}) ; 175.2(\mathrm{CONH}) ; 174.4(\mathrm{CSNHCO}) ; 157.8(\mathrm{C}=\mathrm{NH}) ; 61.9\left(\mathrm{CO}_{2} \mathrm{D}-\mathrm{CH}\right) ; 36.1(\mathrm{C}-7+$ $\left.\mathrm{C}-14+2 \mathrm{xC}_{\text {arginin }}^{5}\right) ; 28.8\left(\mathrm{C}-9+\mathrm{C}-10, \mathrm{C}-11, \mathrm{C}-12+2 \mathrm{xC}^{3}{ }_{\text {arginin }}\right) ; 26.2\left(\mathrm{C}-8+\mathrm{C}-13+2 \mathrm{xC}^{4}\right.$ arginin $)$. Anal. calc. for $\mathrm{C}_{24} \mathrm{H}_{44} \mathrm{~N}_{10} \mathrm{O}_{6} \mathrm{~S}_{2}$ (632.8): C, 45.55; H, 7.01; N, 22.13. Found: C, 45.37; H, 6.93; N, 21.89. MS: m/z (FAB) $633[\mathrm{M}+\mathrm{H}]^{+}$.

Sebacoyl- $N, \boldsymbol{N}$-bis(2-thioureido)propanoic acid (11). From L-alanine $(0.53 \mathrm{~g})$. Yield: 0.96 $(69 \%) ; \mathrm{mp} 257-260{ }^{\circ} \mathrm{C} .{ }^{1} \mathrm{H}$ NMR (DMSO- $\left.d_{6}\right): \delta 3.41\left(\mathrm{~d}, 2 \mathrm{H}, J_{\mathrm{H} 2 \text {-alanin,CH3-alanin })}=3.6 \mathrm{~Hz}\right.$, $2 \mathrm{xH}^{2}$ alanin); $\left.2.25\left(\mathrm{~m}, 4 \mathrm{H},+\mathrm{CH}_{2}-7+\mathrm{CH}_{2}-14\right) ; 1.51\left(\mathrm{~m}, 4 \mathrm{H}, \mathrm{CH}_{2}-8+\mathrm{CH}_{2}-13\right)\right) ; 1.29\left(\mathrm{~m}, 8 \mathrm{H}, \mathrm{CH}_{2}-\right.$ 
9 - $\left.\mathrm{CH}_{2}-12\right) ; 1.24\left(\mathrm{t}, 6 \mathrm{H}, J=7.0 \mathrm{~Hz}, \mathrm{Me}_{\text {alanin }}{ }^{13} \mathrm{C}\right.$ NMR (DMSO-d6): $\delta 186.7$ (C=S); 175.7 $(\mathrm{CSNHCO}) ; 173.0\left(\mathrm{CO}_{2} \mathrm{H}\right) ; 55.9\left(\mathrm{CO}_{2} \mathrm{H}-\mathrm{CH}\right) ; 35.7(\mathrm{C}-7+\mathrm{C}-14) ; 30.2(\mathrm{C}-10+\mathrm{C}-11) ; 28.2(\mathrm{C}-9$ + C-12); 25.5 (C-8 + C-13); 17.3 (Mealanin). Anal. calc. for $\mathrm{C}_{18} \mathrm{H}_{30} \mathrm{~N}_{4} \mathrm{O}_{6} \mathrm{~S}_{2}$ (462.58): C, 46.74; $\mathrm{H}$, 6.54; N, 12.11. Found: C, 46.53; H, 6.47; N, 11.89. MS: m/z (FAB) $463[\mathrm{M}+\mathrm{H}]^{+}$.

Sebacoyl- $\boldsymbol{N}, \boldsymbol{N}$-bis(6-amino-2-thioureido)hexanoic acid (12). From L-lysine $(0.88 \mathrm{~g})$. Yield: $1.40 \mathrm{~g}(81 \%)$; mp230-232 ${ }^{\circ} \mathrm{C} .{ }^{1} \mathrm{H}$ NMR (DMSO- $d_{6}$ ): $\delta 8.02$ (br s., $2 \mathrm{H}, 2 \mathrm{xNH}$ ); 3.67 (t, 2H, $J_{\mathrm{H} 2-}$ lysin,H3-a,b) $=5.5 \mathrm{~Hz}, 2 \mathrm{xH}^{2}$ lysin); $2.76\left(\mathrm{~m} ., 4 \mathrm{H}, 2 \mathrm{xCH}_{2}-\mathrm{NH}_{2}\right) ; 1.78-1.71\left(\mathrm{~m}, 8 \mathrm{H}, 2 \mathrm{xCH}_{2}-3\right.$ lysin $+\mathrm{CH}_{2}-7$ $\left.+\mathrm{CH}_{2}-14\right) ; 1.57$ (m, 4H, 2xCH $2-4$ lysin $) ; 1.45$ (m, 4H, $\left.\mathrm{CH}_{2}-8+\mathrm{CH}_{2}-13\right) ; 1.38$ (m, 4H, $\mathrm{CH}_{2}-9+$ $\left.\mathrm{CH}_{2}-12\right) ; 1.23\left(\mathrm{~m}, 4 \mathrm{H}, \mathrm{CH}_{2}-10+\mathrm{CH}_{2}-11\right) .{ }^{13} \mathrm{C} \mathrm{NMR}$ (DMSO-d $): \delta 187.1(\mathrm{C}=\mathrm{S}) ; 175.8$ $\left(\mathrm{CSNHCO}+\mathrm{CO}_{2} \mathrm{H}\right) ; 63.5\left(\mathrm{CO}_{2} \mathrm{H}-\mathrm{CH}\right) ; 40.0\left(\mathrm{CH}_{2} \mathrm{NH}_{2}\right) ; 38.0(\mathrm{C}-7+\mathrm{C}-14) ; 29.2-27.4\left(\mathrm{C}^{3}\right.$ lysin + $\left.\mathrm{C}^{4}{ }_{\text {lysin }}+\mathrm{C}-9+\mathrm{C}-10+\mathrm{C}-11+\mathrm{C}-12\right) ; 26.1(\mathrm{C}-8+\mathrm{C}-13)$. Anal. calc. for $\mathrm{C}_{24} \mathrm{H}_{44} \mathrm{~N}_{6} \mathrm{O}_{6} \mathrm{~S}_{2}(576.77)$ : C, 49.98; H, 7.69; N, 14.57. Found: C, 49.77; H, 7.58; N, 14.33. MS: m/z (FAB) $577[\mathrm{M}+\mathrm{H}]^{+}$.

Sebacoyl- $\boldsymbol{N}, \boldsymbol{N}$-bis-phthalimide (16). A solution of sebacoyl chloride 1 (2.39 g, $10.0 \mathrm{mmol})$ and phthalimide $13(2.94 \mathrm{~g}, 20 \mathrm{mmol}))$ in acetone $(25 \mathrm{~mL})$ was heated under reflux for $5 \mathrm{~h}$. After cooling the solution was evaporated to dryness to give a crude product followed by washing with water and EtOH. Recrystallization from EtOH afforded 16 (3.82 g, 83\%), mp 215-217 ${ }^{\circ} \mathrm{C} .{ }^{1} \mathrm{H}$ NMR (DMSO-d $): \delta 7.96-7.84(\mathrm{~m}, 8 \mathrm{H}, \mathrm{Ar}-\mathrm{H}) ; 2.19$ (t, $\left.4 \mathrm{H}, J=7.2 \mathrm{~Hz}, \mathrm{CH}_{2}-2+\mathrm{CH}_{2}-9\right) ; 1.52(\mathrm{~m}$, $\left.4 \mathrm{H}, \mathrm{CH}_{2}-3+\mathrm{CH}_{2}-8\right) ; 1.27\left(\mathrm{~m}, 8 \mathrm{H}, \mathrm{CH}_{2}-4-\mathrm{CH}_{2}-7\right) .{ }^{13} \mathrm{C}$ NMR (DMSO-d $): \delta 172.1\left(\mathrm{CH}_{2}-\mathrm{C}=O\right)$; $167.3\left(\mathrm{C}^{\text {phthal. }}=\mathrm{O}\right) ; 134.3,130.1,123.9(\mathrm{Ar}-\mathrm{C}) ; 34.9$ (C-2 + C-9); 28.4 (C-4 - C-7); 24.6 (C-3 + C-8). Anal. calc. for $\mathrm{C}_{26} \mathrm{H}_{24} \mathrm{~N}_{26} \mathrm{O}_{6}$ (460.48): C, 67.82; H, 5.25; N, 6.08. Found: C, 67.61; H, 5.17; $\mathrm{N}$, 5.84. MS: m/z (FAB) $461[\mathrm{M}+\mathrm{H}]^{+}$.

Sebacoyl- $N, N$-bis-methylphthalimide (17). The compound was prepared in the similar manner of preparation of $\mathbf{1 6}$ from hydroxymethyl-phthalimide 14 (3.54 g, $20.0 \mathrm{mmol})$, via $N$ (phthalimido)methylmagnesium bromide 15. Yield: $4.20 \mathrm{~g}(86 \%)$; mp $120-122{ }^{\circ} \mathrm{C}$. ${ }^{1} \mathrm{H}$ NMR (DMSO- $\left.d_{6}\right): \delta$ 7.93-7.86 (m, 8H, Ar-H); 4.96 (s, 4H, 2xCH2-phthal.); 2.17 (t, 4H, J = $7.3 \mathrm{~Hz}$, $\left.\mathrm{CH}_{2}-3+\mathrm{CH}_{2}-10\right) ; 1.47\left(\mathrm{~m}, 4 \mathrm{H}, \mathrm{CH}_{2}-4+\mathrm{CH}_{2}-9\right) ; 1.24\left(\mathrm{~m}, 8 \mathrm{H}, \mathrm{CH}_{2}-5-\mathrm{CH}_{2}-8\right)$. ${ }^{13} \mathrm{C} \mathrm{NMR}$ $\left(\mathrm{DMSO}-d_{6}\right): \delta 174.5\left(\mathrm{CH}_{2}-\mathrm{C}=\mathrm{O}\right) ; 167.4\left(\mathrm{C}^{\text {phthal. }}=\mathrm{O}\right) ; 134.7,131.5,123.7(\mathrm{Ar}-\mathrm{C}) ; 60.1\left(\mathrm{CH}_{2}-\right.$ $\mathrm{C}=\mathrm{O}) ; 38.9(\mathrm{C}-3+\mathrm{C}-10) ; 28.5(\mathrm{C}-5-\mathrm{C}-8) ; 24.4$ (C-4 + C-9). Anal. calc. for $\mathrm{C}_{28} \mathrm{H}_{28} \mathrm{~N}_{2} \mathrm{O}_{6}$ (488.53): C, 68.84; H, 5.78; N, 5.73. Found: C, 68.62; H, 5.69; N, 5.50. MS: m/z (FAB) 489 $[\mathrm{M}+\mathrm{H}]^{+}$.

Sebacoyl- $N, N$-bis-methyl-(3-methyl-2-imino-thiazol-3-yl)butanoate (18). To a stirred solution of $5(0.55 \mathrm{~g}, 1.0 \mathrm{mmol})$ in dry acetone $(20 \mathrm{~mL})$ was added $\mathrm{Et}_{3} \mathrm{~N}(1.0 \mathrm{mmol})$, followed by a dropwise addition of a bromine solution $(1.0 \mathrm{mmol})$ in acetone $(10 \mathrm{~mL})$. The reaction mixture was stirred at room temperature for $2 \mathrm{~h}$, then the mixture was evaporated to dryness to give the desired product, which were recrystallized from EtOH to afford 18 (0.41 g, 65\%), mp 252$254{ }^{\circ} \mathrm{C} .{ }^{1} \mathrm{H}$ NMR (DMSO-d $): \delta 5.87$ (s, 2H, $2 \mathrm{xH}^{5}$ thiazol); $3.68\left(\mathrm{~s}, 3 \mathrm{H}, \mathrm{CO}_{2} \mathrm{Me}\right.$ ); 3.48 (dd, 2H, $\left.J_{2,3 \text { (valin })}=7.3 \mathrm{~Hz}, 2 \mathrm{xH}^{2}{ }_{\text {valin }}\right) ; 2.75\left(\mathrm{~m}, 2 \mathrm{H}, 2 \mathrm{xH}^{3}{ }_{\text {valin }}\right) ; 2.13\left(\mathrm{~m}, 4 \mathrm{H}, 2 \mathrm{xCOCH} \mathrm{H}_{2 \text { sebacoyl }}\right) ; 1.70(\mathrm{~s}, 6 \mathrm{H}$, $2 \mathrm{xC}^{4}$ triazol $\left.-\mathrm{CH}_{3}\right) ; 1.55\left(\mathrm{~m}, 4 \mathrm{H}, 2 \mathrm{xCOCH}_{2} \mathrm{CH}_{2 \text { sebacoyl }}\right) ; 1.30\left(\mathrm{~m}, 8 \mathrm{H}, 4 \mathrm{xCH}_{2 \text { sebacoyl }}\right) ; 1.07$ (m, $12 \mathrm{H}$, $\left.4 \mathrm{xCH}_{3 \text { valin }}\right) .{ }^{13} \mathrm{C} \mathrm{NMR}\left(\mathrm{DMSO}-d_{6}\right): \delta 173.1\left(2 \mathrm{xC}^{\text {sebacoyl }}=\mathrm{O}\right) ; 171.5\left(2 \mathrm{x} C \mathrm{O}_{2} \mathrm{Me}\right) ; 163.8(\mathrm{C}=\mathrm{N})$; $132.9\left(\mathrm{C}^{4}\right.$ thiazol $) ; 100.1\left(\mathrm{C}^{5}\right.$ thiazol $) ; 60.5\left(2 \mathrm{xCO}_{2} \mathrm{Me}-\mathrm{CH}\right) ; 52.4\left(2 \mathrm{xCO}_{2} \mathrm{Me}\right) ; 34.1\left(2 \mathrm{xCOCH}_{2 \text { sebacoyl }}\right)$; 


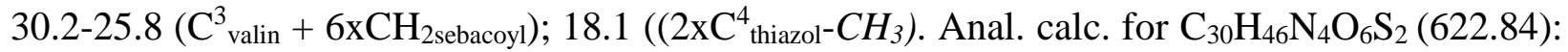
C, 57.85; H, 7.44; N, 9.00. Found: C, 57.67; H, 7.34; N, 8.82. MS: m/z (FAB) $623[\mathrm{M}+\mathrm{H}]^{+}$.

Sebacoyl- $N, N$-bis-(3-mercapto-2-(4-methyl-2-imino-thiazol-3-yl)propanoic acid (19). The compound was prepared in the similar manner of preparation of 18 from $7(0.53 \mathrm{~g}, 1.0 \mathrm{mmol})$. Yield: $0.43 \mathrm{~g}(71 \%)$; mp $243-246{ }^{\circ} \mathrm{C} .{ }^{1} \mathrm{H}$ NMR (DMSO- $\left.d_{6}\right): \delta 5.92$ (s, $2 \mathrm{H}, 2 \mathrm{xH}^{5}$ thiazol); 3.90 (dd, $\left.2 \mathrm{H}, J_{\mathrm{H} 2 \text {-cystein,H3-a) }}=7.0 \mathrm{~Hz}, J_{\mathrm{H} 2 \text {-cystein,H3-b) }}=13.9 \mathrm{~Hz} 2 \mathrm{xH}^{2}{ }_{\text {cystein }}\right) ; 3.10$ (m., $4 \mathrm{H}, 2 \mathrm{xH}^{3 \mathrm{a}}$ cystein + $2 \mathrm{xH}^{3 \mathrm{~b}}$ cystein $) ; 2.38\left(\mathrm{~m}, 4 \mathrm{H},+2 \mathrm{xCOCH}_{2 \text { sebacoyl }}\right) ; 1.68\left(\mathrm{~s}, 6 \mathrm{H}, 2 \mathrm{xC}^{4}{ }_{\text {triazol }}-\mathrm{CH}_{3}\right) ; 1.58(\mathrm{~m}, 4 \mathrm{H}$, $2 \mathrm{xCOCH}_{2} \mathrm{CH}_{2 \text { sebacoyl }) ;} \quad 1.28 \quad\left(\mathrm{~m}, \quad 8 \mathrm{H}, \quad 4 \mathrm{xCH}_{2 \text { sebacoyl }}\right) .{ }^{13} \mathrm{C} \quad \mathrm{NMR} \quad\left(\mathrm{DMSO}-d_{6}\right): \quad \delta \quad 173.3$ $\left(2 \times \mathrm{C}^{\text {sebacoyl }}=\mathrm{O}\right) ; 163.6 \quad(\mathrm{C}=\mathrm{N}) ; 132.7 \quad\left(\mathrm{C}_{\text {thiazol }}^{4}\right) ; 99.8 \quad\left(\mathrm{C}^{5}\right.$ thiazol $) ; 62.7 \quad\left(\mathrm{CO}_{2} \mathrm{H}-\mathrm{CH}\right) ; 31.8$ $\left(2 \mathrm{xCOCH} \mathrm{Csebacoyl}_{2}\right) ; 28.5\left(4 \mathrm{xCH}_{2 \text { sebacoyl }}\right) ; 25.3\left(2 \mathrm{xCOCH}_{2} \mathrm{CH}_{2 \text { sebacoyl }}\right) ; 24.1 \quad\left(2 \mathrm{xCH}_{2} \mathrm{SH}\right) ; 18.2$

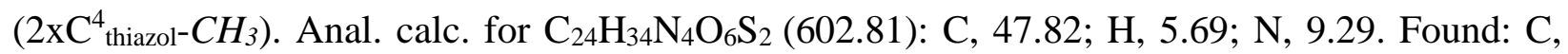
47.61; H, 5.59; N, 9.01. MS: m/z (FAB) $603[\mathrm{M}+\mathrm{H}]^{+}$.

\section{Acknowledgments}

We thank Mr. U. Haunz of chemistry department, University of Konstanz, Germany for the NMR experiments.

\section{References}

1. De Clercq, E. Med. Chem. Res. 2004, 13, 439.

2. Barbaro, G.; Scozzafava, A.; Mastrolorenzo, A.; Supuran, C. T. Curr. Pharm. Des. 2005, 11, 1805.

3. Koup, R. A.; Merluzzi, V. J.; Hargrave, J. L. J. Infect. Dis. 1991, 163, 966.

4. Freimuth, W. W. Adv. Exp. Med. Biol. 1996, 394, 279.

5. Young, S. D.; Britcher, S. F.; Tran, L. O.; Payne, L. S.; Lumma, W. C.; Lyle, T. A.; Huff, J. R.; Anderson, P. S.; Olsen, D. B.; Carroll, S. S.; Pettibone, D. J.; Obrien, J. A.; Ball, R. G.; Balani, S. K.; Lin, J. H.; Chen, I. W.; Schleif, W. A.; Sardana, V. V.; Long, W. J.; Byrnes, V. W.; Emini, E. A. Antimicrob. Agents Chemother. 1995, 39, 2602.

6. Wainberg, M. A.; Sawyer, J. P.; Montaner, J. S.; Murphy, R. L.; Kuritzkes, D. R.; Raffi, F. Antiviral Ther. 2005, 10, 13.

7. Imamichi, T. Curr. Pharm. Des. 2004, 10, 4039.

8. Amajaour, H. A. S.; Al-Soud, Y. A.; Al-Sa'doni, H.; Al-Masoudi, N. A. Z. Naturforsch. (J. Chem. Sci). 2007, 62b, 523.

9. Al-Masoudi, N. A.; Al-Soud, Y. A.; Kalogerakis, A.; De Clercq, E.; Paneccoque, C. Chem. Biodiver. 2006, 3, 515.

10. Y. A. Al-Soud, N. A. Al-Masoudi, E. De Clercq and C. Paneccoque, Heteroatom Chem. 2007, 4, 333. 
11. Al-Soud, Y. A.; Al-Masoudi, N. A.; De Clercq, E.; Paneccoque, C. Antiviral Chem. Chemother. 2007, 18, 191.

12. Al-Soud, Y. A.; Al-Masoudi, N. A.; Gh. Hassan, H.; Gh.; De Clercq, E.; Pannecouque, C. Acta Pharm. 2007, 57, 379.

13. Ahgren, C.; Backro, K.; Bell, F. W.; Cantrell, A. S.; Clemens, M.; Colacino, J.; Deeter, M. J. B.; Engelhardt, J. A.; Jaskunas, S. R.; Johansson, N. G.; Jordan, C. L.; Kasher, J. S.; Kinnick, M. D.; Lind, P.; Lopez, C.; Morin, J. M.; Muesing, M. A.; Noreen, R.; Oberg, B.; Paget, C. J.; Palkowitz, J. A.; Parrish, C.; Pranc, P.; Rippy, M. K.; Rydergard, C.; Sahlberg, C.; Swanson, S.; Ternansky, R.; Unge, J.; Vasileff, T. R. T.; Vrang, L.; West, S. J.; Zhang, H.; Zhou, X. X. Antimicrob. Agents Chemother. 1995, 39, 1329.

14. Heinisch, G.; Matuszczak, B.; Pachler, S.; Rakowitz, D. Antivir. Chem. Chemother. 1997, 8, 443.

15. Ren, J.; Diprose, J.; Warren, J.; Esnouf, R. M.; Bird, L. E.; Ikemizu, S.; Slater, M.; Milton, J.; Balzarini, J.; Stuart, D. I; Stammers, D. K. J. Biol. Chem. 2000, 275, 5633.

16. Ahgren, C.; Backro, K.; Bell, F. W.; Cantrell, S.; Clemens, M.; Colacino, J. M.; Deeter, M. J. B.; Engelhardt, J. A.; Hogberg, M.; Jaskunas, S. R. Antimicrob. Agents Chemother. 1995, 39, 1329.

17. Uckun, F. M.; Venkatachalam, T. K. United States Patent 2005, 6960606.

18. Fathalla, W.; Pazdera, P. Arkivoc 2007, (i), 236.

19. Fathalla, W.; Ali, I. A. I. Heteroatom Chem. 2007, 18, 637.

20. Fathalla, W.; El Rayes, S. M.; Ali, I. A. I. Arkivoc 2007, (xvi), 173.

21. El Rayes, S. M.; Ali, I. A. I.; Fathalla, W. Arkivoc 2008, (xi), 86.

22. Fathalla, W.; Pazdera, P. Arkivoc 2007, (i), 236.

23. 23.. Fathalla, W. Arkivoc 2008, (xii), 245.

24. Ali, I. A. I.; Al-Masoudi, I. A.; Saeed, B.; Al-Masoudi; N. A.; La Colla, P. Heteroatom Chem. 2005, 16, 148.

25. Al-Masoudi, N. A.; Al-Masoudi, I. A.; Ali, I . A. I.; Saeed, B.; La Colla, P. Heteroatom Chem. 2005, 16, 576.

26. Ali, I. A. I.; Al-Masoudi, I. A.; Al-Soud, Y. A.; Saeed, B.; Al-Masoudi, N. A.; La Colla, P.Acta Pharm. 2006, 56, 175.

27. Hamad, N.S.; Al-Haidari, N. H.; Al-Masoudi, I. A.; Sabri, M.; Sabri, L.; Al-Masoudi, N. A. Arch. Pharmazie- life Science 2010, in press.

28. Masllorens, J.; Pla-Quintana, A.; Parella,T.; Roglans, A. Arkivoc 2010, (ii), 203.

29. Koenig, K.-H.; Kuge, M.; Kaul, L.; Pletsch, H.-J. Chem. Ber. 1987, 120, 1251.

30. Kabbani, A. T.; Ramadan, H.; Hammud, H. H.; Ghannoum, A. M.; Mouneimne, Y. J. Uni. Chem. Techn. Metal. 2005, 40, 339.

31. Al-Masoudi, N A.; Al-Soud, Y. A.; Geyer, A. Spectroscopy Lett. 1998, 31, 1031.

32. Bax, A.; Griffey, R. H.; Hawkins, B. L. Correlation J. Magn. Reson. 1983, 55, 301

33. Willker, W.; Leibfritz, D.; Kerssebaum, R.; Bermel, W. Mag. Reson. 1993, Chem. 31, 287.

34. Hong, S. I.; Kim, C. K.; Kim, Y. J. Bull. Korean Chem. Soc. 1983, 4, 171. 
35. R. Pauwels, J. Balzarini, M. Baba, R. Snoeck, D. Schols, P. Herdewijn, J. Desmyter, E. De Clercq, J. Virol. Methods 1988, 20, 309.

36. K. D. Hargrave, J. R. Proudfoot, K. G. Grozinger, E. Cullen, S. R. Kapadia, U. R. Patel, V. U. Fuchs, S. C. Mauldin, J. Vitous, M. L. Behnke, J. M. Klunder, K. Pal, J. W. Skiles, D. W. McNeil, J. M. Rose, G. C. Chow, M. T. Skoog, J. C. Wu, G. Schmidt, W. W. Engel, W. G. Eberlein, T. D. Saboe, S. J. Campbell, A. S. Rosenthal, J. Adam, J. Med. Chem. 1991, 34, 2231.

37. Mitsuya, H.; Weinhold, K. J.; Furman, P. A.; St. Clair, M. H.; Lehrmann, S. N.; Gallo, R. C.; Bolognesi, D.; Barry, D.W.; Broder, S. Proc Natl Acad Sci USA 1985, 82, 7096. 